\title{
Group splittings and integrality of traces
}

\author{
Ioannis Emmanouil*
}

\begin{abstract}
In this paper, we elaborate on Connes' proof of the integrality of the trace conjecture for free groups, in order to show that any action of a group $G$ on a tree leads to a similar integrality assertion concerning the trace on the group algebra $\mathbb{C} G$, which is associated with the set of group elements that stabilize a vertex.
\end{abstract}

Mathematics Subject Classification (2000). 19A31, 20E08.

Keywords. Traces, $K_{0}$-group, group actions on trees.

\section{Introduction}

Given a torsion-free group $G$, the integrality of the trace conjecture is the assertion that the image of the additive map

$$
\tau_{*}: K_{0}\left(C_{r}^{*} G\right) \longrightarrow \mathbb{C},
$$

which is induced by the canonical trace $\tau$ on the reduced $C^{*}$-algebra $C_{r}^{*} G$ of $G$, is the group $\mathbb{Z}$ of integers. Some evidence for the validity of that conjecture is provided by Zalesskii's theorem [18], which states that for any group $G$ (possibly with torsion) the values of $\tau_{*}$ on $\mathrm{K}$-theory classes that come from the group algebra $\mathbb{C} G$ are rational. By a standard argument, the integrality of the trace conjecture can be shown to imply the triviality of idempotents in $C_{r}^{*} G$. In the case where $G$ is a free group, Connes proved in [5, §IV.5] the integrality of the trace conjecture by using a free action of $G$ on a tree and the associated representations of $C_{r}^{*} G$ on the Hilbert space $\ell^{2} V$, where $V$ is the set of vertices of the tree. We also note that, in the case of a torsion-free abelian group $G$, the integrality of the trace conjecture is an immediate consequence of the connectedness of the dual group $\widehat{G}$ (cf. [16, Theorem 2]).

In the case where the group $G$ has non-trivial torsion elements, Baum and Connes had conjectured in [3] that the image of $\tau_{*}$ is the subgroup of $\mathbb{Q}$ generated by the inverses of the orders of the finite subgroups of $G$. This latter conjecture was disproved by Roy [14]. Subsequently, Lück [10] formulated a modified version of that

\footnotetext{
${ }^{*}$ Research funded by the University of Athens Special Research Account, grant 70/4/6413.
} 
conjecture, according to which the image of $\tau_{*}$ is contained in the subring of $\mathbb{Q}$ generated by the inverses of the orders of the finite subgroups of $G$, and showed that this is indeed the case if the so-called Baum-Connes assembly map is surjective.

In this paper, we are interested in traces defined on the group algebra $\mathbb{C} G$ and examine integrality properties of the induced additive maps on the K-theory group $K_{0}(\mathbb{C} G)$. If $S \subseteq G$ is a subset closed under conjugation, then the linear map (partial augmentation)

$$
\tau_{S}: \mathbb{C} G \longrightarrow \mathbb{C},
$$

which is defined by letting $\tau_{S}\left(\sum_{g \in G} a_{g} g\right)=\sum_{g \in S} a_{g}$ for any element $\sum_{g \in G} a_{g} g \in$ $\mathbb{C} G$, is a trace. As such, it induces an additive map

$$
\left(\tau_{S}\right)_{*}: K_{0}(\mathbb{C} G) \longrightarrow \mathbb{C} .
$$

In the special case where $S=G$, the additive map $\left(\tau_{S}\right)_{*}$ is that induced by the augmentation homomorphism $\mathbb{C} G \rightarrow \mathbb{C}$. The map $\left(\tau_{S}\right)_{*}$ is then referred to as the homological (or naive) rank and its image is the group $\mathbb{Z}$ of integers (cf. [4, Chapter IX, Exercise 2.5]). On the other hand, if $S=\{1\}$, then the map $\left(\tau_{S}\right)_{*}$ is the Kaplansky rank, whose values are rational in view of Zaleskii's theorem [18]. In fact, if $G$ is torsion-free then a weak version of Bass' trace conjecture [2] asserts that the Kaplansky rank coincides with the homological rank; if this is true, then we must have $\operatorname{im}\left(\tau_{S}\right)_{*}=\mathbb{Z}$ in this case as well.

We can now state our main result.

Theorem. Let $G$ be the fundamental group of a connected graph of groups with vertex groups $\left(G_{v}\right)_{v}$ and edge groups $\left(G_{e}\right)_{e}$, and consider the subset $S \subseteq G$ which consists of the conjugates of all elements of the set $\bigcup_{v} G_{v}$. Then, $\operatorname{im}\left(\tau_{S}\right)_{*}=\mathbb{Z}$.

Equivalently, we may state the result above in terms of the universal trace defined by Hattori and Stallings, as follows: If $x \in K_{0}(\mathbb{C} G)$ is a K-theory class and $r_{[g]}(x) \in \mathbb{C}$ the coefficient of the Hattori-Stallings rank $r_{\mathrm{HS}}(x)$ that corresponds to the conjugacy class $[g]$ of an element $g \in G$, then $\sum_{[g] \in[S]} r_{[g]}(x) \in \mathbb{Z}$, where $[S]$ is the set of conjugacy classes of the elements of $S$.

We observe that our integrality result would follow immediately if one could show that the additive map

$$
\bigoplus_{v} K_{0}\left(\mathbb{C} G_{v}\right) \longrightarrow K_{0}(\mathbb{C} G),
$$

which is induced by the inclusion of the vertex groups $G_{v}$ into $G$, is surjective. Indeed, for any vertex $v$ the composition

$$
K_{0}\left(\mathbb{C} G_{v}\right) \longrightarrow K_{0}(\mathbb{C} G) \stackrel{\left(\tau_{S}\right)_{*}}{\longrightarrow} \mathbb{C}
$$

is the additive map induced by the restriction of the trace $\tau_{S}$ on $\mathbb{C} G_{v}$ (cf. Remark 1.1 (ii) below). But $S$ contains $G_{v}$ and hence the restriction of $\tau_{s}$ on $\mathbb{C} G_{v}$ 
is the augmentation homomorphism $\mathbb{C} G_{v} \rightarrow \mathbb{C}$. Therefore, the composition (2) is the homological rank associated with $G_{v}$; in particular, its image is the group $\mathbb{Z}$ of integers. In view of the assumed surjectivity of (1), we conclude that the image of $\left(\tau_{S}\right)_{*}$ is the group $\mathbb{Z}$ as well.

We note that if $G$ is the fundamental group of a graph of groups as above, then any finite subgroup $H \subseteq G$ is contained in a conjugate of $G_{v}$ for some vertex $v$ of the graph (cf. [15, Chapitre I, Exemple 6.3.1]). Since conjugation by any element of $G$ induces the identity map on $K_{0}(\mathbb{C} G)$, we conclude that the map (1) is surjective if this is the case for the additive map

$$
\bigoplus_{H} K_{0}(\mathbb{C} H) \longrightarrow K_{0}(\mathbb{C} G) .
$$

Here, the direct sum is over all finite subgroups $H$ of $G$ and the map is induced by the inclusion of the $H$ 's into $G$. In particular, it follows that the map (1) is surjective if the so-called isomorphism conjecture for $K_{0}(\mathbb{C} G)$ holds (cf. [11, Conjecture 9.40]). On the other hand, we may consider the special case where the graph has one edge $e$ and two distinct vertices $v_{1}$ and $v_{2}$. In that case, $G$ is the amalgamated free product $G_{v_{1}} \star_{G_{e}} G_{v_{2}}$ and a sufficient condition that guarantees the surjectivity of (1) has been given by Waldhausen (see the discussion following [17, Corollary 11.5]).

From the point of view presented above, there is a formal resemblance between our result and those described in [1] and [12], where it is proved that a certain statement is true for $G$ if it is true for all $G_{v}$ 's.

We also observe that our integrality result would follow if one could show that the group $G$ satisfies (the strong version of) Bass' trace conjecture [2]. Indeed, the latter conjecture asserts that for any $x \in K_{0}(\mathbb{C} G)$ the coefficient $r_{[g]}(x)$ of the HattoriStallings rank $r_{\mathrm{HS}}(x)$ vanishes if $g \in G$ is an element of infinite order. Since $S$ contains all group elements of finite order (as we have already noted above), this would imply that $\left(\tau_{S}\right)_{*}(x)$ is the homological rank of $x$; in particular, it would follow that $\left(\tau_{S}\right)_{*}(x) \in \mathbb{Z}$.

The contents of the paper are as follows: In Section 1, we recall certain wellknown facts concerning traces on an algebra and the induced additive maps on the $K_{0}$-group. In the following section, we consider a group $G$ acting on a tree and examine certain representations of the group algebra $\mathbb{C} G$. In Section 3, we prove our main result and explicit the integrality assertions in the special cases where $G$ is an amalgamated free product or an HNN extension. Finally, in the last section, we examine whether our integrality result can be extended from the group algebra $\mathbb{C} G$ to the reduced group $C^{*}$-algebra $C_{r}^{*} G$ of $G$.

It is a pleasure to thank M. Karoubi, P. Papasoglu, O. Talelli and M. Wodzicki for some helpful comments, as well as the referee, whose suggestions improved the article. 


\section{Traces and the $K_{0}$-group}

Let $R$ be a unital ring, $V$ an abelian group and $\tau: R \rightarrow V$ a trace, i.e. an additive map which vanishes on the commutators $x y-y x$ for all $x, y \in R$. Then, for any positive integer $n$ the map

$$
\tau_{n}: \mathrm{M}_{n}(R) \longrightarrow V,
$$

which is defined by letting $\tau_{n}(A)=\sum_{i=1}^{n} \tau\left(a_{i i}\right)$ for any matrix $A=\left(a_{i j}\right)_{i, j} \in$ $\mathrm{M}_{n}(R)$, is a trace as well. These traces induce an additive map

$$
\tau_{*}: K_{0}(R) \longrightarrow V,
$$

by mapping the K-theory class of any idempotent matrix $E \in \mathrm{M}_{n}(R)$ onto $\tau_{n}(E)$.

Remarks 1.1. (i) Let $R$ be a ring and $f: V \rightarrow V^{\prime}$ an abelian group homomorphism. We consider a trace $\tau: R \rightarrow V$ and the $V^{\prime}$-valued trace $f \circ \tau$ on $R$. Then, the induced additive map $(f \circ \tau)_{*}: K_{0}(R) \rightarrow V^{\prime}$ is the composition

$$
K_{0}(R) \stackrel{\tau_{*}}{\longrightarrow} V \stackrel{f}{\longrightarrow} V^{\prime},
$$

where $\tau_{*}: K_{0}(R) \rightarrow V$ is the additive map induced by the trace $\tau$.

(ii) Let $\varphi: R \rightarrow S$ be a ring homomorphism and $V$ an abelian group. We consider a trace $\tau: S \rightarrow V$ and the $V$-valued trace $\tau \circ \varphi$ on $R$. Then, the induced additive $\operatorname{map}(\tau \circ \varphi)_{*}: K_{0}(R) \rightarrow V$ is the composition

$$
K_{0}(R) \stackrel{K_{0}(\varphi)}{\longrightarrow} K_{0}(S) \stackrel{\tau_{*}}{\longrightarrow} V,
$$

where $\tau_{*}: K_{0}(S) \rightarrow V$ is the additive map induced by the trace $\tau$.

(iii) Let $R$ be a ring and $[R, R]$ the additive subgroup of it generated by the commutators $x y-y x, x, y \in R$. Then, the quotient map $p: R \rightarrow R /[R, R]$ is the universal trace defined on $R$ and induces the Hattori-Stallings rank map

$$
r_{\mathrm{HS}}: K_{0}(R) \longrightarrow R /[R, R]
$$

see also [4, Chapter IX, §2].

(iv) In the special case where $R=\mathbb{C} G$ is the group algebra of a group $G$, the quotient group $R /[R, R]=\mathbb{C} G /[\mathbb{C} G, \mathbb{C} G]$ is a complex vector space with basis the set $\mathfrak{C}(G)$ of conjugacy classes of elements of $G$. If $[g] \in \mathfrak{C}(G)$ is the conjugacy class of an element $g \in G$, then the linear functional (partial augmentation) $\sum_{h \in G} a_{h} h \mapsto \sum_{h \in[g]} a_{h}, \sum_{h \in G} a_{h} h \in \mathbb{C} G$, is a trace and hence induces an additive map

$$
r_{[g]}: K_{0}(\mathbb{C} G) \longrightarrow \mathbb{C} .
$$

These maps determine the Hattori-Stallings rank of any K-theory class $x \in K_{0}(\mathbb{C} G)$, since we have $r_{\mathrm{HS}}(x)=\sum_{[g] \in \mathbb{C}(G)} r_{[g]}(x)[g] \in \mathbb{C} G /[\mathbb{C} G, \mathbb{C} G]$. 
We now consider a non-unital ring $I$ and let $I^{+}$be the associated unital ring. Here, $I^{+}=I \oplus \mathbb{Z}$ as an abelian group, whereas the product of any two elements $(x, n),(y, m) \in I^{+}$is equal to $(x y+n y+m x, n m) \in I^{+}$. The $K_{0}$-group of $I$ is defined by means of the split extension

$$
0 \longrightarrow I \longrightarrow I^{+} \stackrel{\pi}{\longrightarrow} \mathbb{Z} \longrightarrow 0,
$$

where $\pi$ is the projection $(x, n) \mapsto n,(x, n) \in I^{+}$. More precisely, $K_{0}(I)$ is the kernel of the induced additive map $K_{0}(\pi): K_{0}\left(I^{+}\right) \rightarrow K_{0}(\mathbb{Z})$. Let $\tau: I \rightarrow V$ be a $V$-valued trace on $I$; by this, we mean that $\tau$ is an additive map which vanishes on the commutators $x y-y x$ for all $x, y \in I$. Then, $\tau$ extends to an additive map $\tau^{+}$on the associated unital ring $I^{+}$, by letting $\tau^{+}(0,1)=0$; in fact, $\tau^{+}$is a trace. The induced additive map $\tau_{*}: K_{0}(I) \rightarrow V$ is defined as the restriction of $\left(\tau^{+}\right)_{*}: K_{0}\left(I^{+}\right) \rightarrow V$ to the subgroup $K_{0}(I) \subseteq K_{0}\left(I^{+}\right)$.

Example 1.2. Let $U$ be a complex vector space with basis $\left(\xi_{i}\right)_{i}$ and $L(U)$ the algebra of linear endomorphisms of $U$. We consider the ideal $\mathfrak{F} \subseteq L(U)$ consisting of those endomorphisms of $U$ that have finite rank. Then, for any $a \in \mathfrak{F}$ the family of complex numbers $\left(\left[a\left(\xi_{i}\right), \xi_{i}^{*}\right]\right)_{i}$ has finite support. Here, we denote for all $i$ by $\xi_{i}^{*}$ the linear functional on $U$ which maps $\xi_{i}$ onto 1 and vanishes on $\xi_{j}$ for $j \neq i$, whereas [, ] denotes the standard pairing between $U$ and its dual. Moreover, the map

$$
\operatorname{Tr}: \mathfrak{F} \longrightarrow \mathbb{C},
$$

which is defined by letting $\operatorname{Tr}(a)=\sum_{i}\left[a\left(\xi_{i}\right), \xi_{i}^{*}\right]$ for all $a \in \mathfrak{F}$, does not depend upon the choice of the basis $\left(\xi_{i}\right)_{i}$ and vanishes on the elements of the form $a b-b a$, $a \in \mathfrak{F}, b \in L(U)$. In particular, Tr is a trace on $\mathfrak{F}$. In view of the Morita invariance and the continuity of the functor $K_{0}$ (cf. [13, Chapter $\left.1, \S 2\right]$ ), the induced additive map

$$
\operatorname{Tr}_{*}: K_{0}(\mathfrak{F}) \longrightarrow \mathbb{C}
$$

identifies $K_{0}(\mathfrak{F})$ with the subgroup $\mathbb{Z} \subseteq \mathbb{C}$. More generally, let $R$ be a complex algebra and consider a linear trace $\tau$ on $R$ with values in a complex vector space $V$. Then, the linear map

$$
\operatorname{Tr} \otimes \tau: \mathfrak{F} \otimes R \longrightarrow \mathbb{C} \otimes V \simeq V,
$$

which is defined by letting $a \otimes x \mapsto \operatorname{Tr}(a) \tau(x)$ for all elementary tensors $a \otimes x \in$ $\mathfrak{F} \otimes R$, is also a trace. Moreover, the induced additive map

$$
(\operatorname{Tr} \otimes \tau)_{*}: K_{0}(\mathfrak{F} \otimes R) \longrightarrow V
$$

is identified with the additive map

$$
\tau_{*}: K_{0}(R) \longrightarrow V,
$$

which is induced by $\tau$, in view of the Morita isomorphism $K_{0}(\mathfrak{F} \otimes R) \simeq K_{0}(R)$. 
A proof of the following result may be found in [9, Proposition 1.44].

Proposition 1.3. Let $\varphi, \psi: A \rightarrow B$ be two homomorphisms of non-unital rings and $I \subseteq B$ an ideal such that $\psi(a)-\varphi(a) \in I$ for all $a \in A$. We consider an abelian group $V$ and an additive map $\tau: I \rightarrow V$ that vanishes on elements of the form $x y-y x$ for all $x \in I$ and $y \in B$; in particular, $\tau$ is a trace on $I$. Let $t: A \rightarrow V$ be the additive map which is defined by letting $t(a)=\tau(\psi(a)-\varphi(a))$ for all $a \in A$. Then:

(i) The map $t$ is a trace on A.

(ii) The image of the additive map $t_{*}: K_{0}(A) \rightarrow V$ is contained in the image of the additive map $\tau_{*}: K_{0}(I) \rightarrow V$.

\section{Trees and group actions}

Let $X$ be a graph and denote by $V, E^{\text {or }}$ the set of vertices and oriented edges of it respectively. A path on $X$ is a finite sequence $\left(e_{1}, \ldots, e_{n}\right)$ of oriented edges such that the terminus $v_{i}$ of $e_{i}$ is the origin of $e_{i+1}$ for all $i=1, \ldots, n-1$. We say that a path as above has origin $v_{0}$ equal to the origin of $e_{1}$, terminus $v_{n}$ equal to the terminus of $e_{n}$ and passes through the vertices $v_{1}, \ldots, v_{n-1}$. The path is reduced if there is no $i$ such that $e_{i+1}$ is equal to the reverse edge of $e_{i}$. The graph $X$ is a tree if for any two vertices $v, v^{\prime} \in V$ with $v \neq v^{\prime}$ there is a unique reduced path with origin $v$ and terminus $v^{\prime}$; this path, denoted by $\left[v, v^{\prime}\right]$, is called the geodesic joining $v$ and $v^{\prime}$.

Let $X$ be a tree and denote by $E$ the corresponding set of un-oriented edges. It is well known that the number of vertices exceeds the number of un-oriented edges by one. More precisely, having fixed a vertex $v_{0} \in V$, we consider for any $v \in V \backslash\left\{v_{0}\right\}$ the geodesic $\left[v_{0}, v\right]=\left(e_{1}, \ldots, e_{n}\right)$ and define the map

$$
\lambda: V \backslash\left\{v_{0}\right\} \longrightarrow E,
$$

by letting $\lambda(v)$ be the un-oriented edge associated with the oriented edge $e_{n}$. The proof of the next result is straightforward.

Lemma 2.1. Let $X$ be a tree and fix a vertex $v_{0} \in V$.

(i) The map $\lambda$ defined above is bijective.

(ii) For another vertex $v_{0}^{\prime} \in V$ consider the corresponding map $\lambda^{\prime}: V \backslash\left\{v_{0}^{\prime}\right\} \rightarrow E$. If the geodesic $\left[v_{0}, v_{0}^{\prime}\right]$ passes through the vertices $v_{1}, \ldots, v_{n-1}$, then we have $\lambda(v)=\lambda^{\prime}(v)$ for all vertices $v \in V \backslash\left\{v_{0}, v_{1}, \ldots, v_{n-1}, v_{0}^{\prime}\right\}$.

Let $\alpha$ be an automorphism of the tree $X$ and denote by $\alpha_{V}$ (resp. $\alpha_{E}$ ) the corresponding bijection of the set of vertices (resp. edges) of $X$. We fix a vertex $v_{1} \in V$ 
and consider the associated bijection $\lambda_{1}: V \backslash\left\{v_{1}\right\} \rightarrow E$. We also consider the vertex $v_{2}=\alpha_{V}\left(v_{1}\right) \in V$ and the associated bijection $\lambda_{2}: V \backslash\left\{v_{2}\right\} \rightarrow E$. Then, it is easily seen that

$$
\alpha_{E} \circ \lambda_{1}=\lambda_{2} \circ \alpha_{V}^{\prime},
$$

where $\alpha_{V}^{\prime}$ denotes the restriction of $\alpha_{V}$ to the subset $V \backslash\left\{v_{1}\right\} \subseteq V$. The automorphism $\alpha$ is said to have no inversions if there is no edge $e \in E^{\text {or }}$ such that $\alpha(e)$ is the reverse edge of $e$.

Proposition 2.2. Let $X$ be a tree, $v_{0} \in V$ a vertex and $\lambda: V \backslash\left\{v_{0}\right\} \rightarrow E$ the associated bijection. We consider a group $G$ acting on $X$ and fix an element $g \in G$.

(i) If $g \cdot v_{0}=v_{0}$, then we have $g \cdot \lambda(v)=\lambda(g \cdot v)$ for all $v \in V \backslash\left\{v_{0}\right\}$.

(ii) If $g \cdot v_{0} \neq v_{0}$ and the geodesic $\left[v_{0}, g^{-1} \cdot v_{0}\right]$ passes through the vertices $v_{1}, \ldots, v_{n-1}$, then $g \cdot \lambda(v)=\lambda(g \cdot v)$ for all $v \in V \backslash\left\{v_{0}, v_{1}, \ldots, v_{n-1}, g^{-1} \cdot v_{0}\right\}$.

Proof. We consider the vertex $g^{-1} \cdot v_{0}$ and let

$$
\lambda^{\prime}: V \backslash\left\{g^{-1} \cdot v_{0}\right\} \longrightarrow E
$$

be the associated bijection. The element $g \in G$ induces an automorphism of the tree $X$, which maps the vertex $g^{-1} \cdot v_{0}$ onto $v_{0}$, and hence Equation (3) above implies that $g \cdot \lambda^{\prime}(v)=\lambda(g \cdot v)$ for all $v \in V \backslash\left\{g^{-1} \cdot v_{0}\right\}$. This completes the proof in the case where $g \cdot v_{0}=v_{0}$, since we then have $\lambda=\lambda^{\prime}$. If $g \cdot v_{0} \neq v_{0}$, the proof is finished by invoking Lemma 2.1 (ii), which implies that $\lambda(v)=\lambda^{\prime}(v)$ for all vertices $v \in V \backslash\left\{v_{0}, v_{1}, \ldots, v_{n-1}, g^{-1} \cdot v_{0}\right\}$.

Let $X$ be a graph and consider the sets $V, E$ of vertices and (un-oriented) edges of $X$ respectively and the complex vector spaces $\mathbb{C}^{(V)}=\bigoplus_{v \in V} \mathbb{C} \cdot \xi_{v}$ and $\mathbb{C}^{(E)}=$ $\bigoplus_{e \in E} \mathbb{C} \cdot \xi_{e}$. If $G$ is a group acting on $X$, then for any element $g \in G$ we denote by

$$
\varrho_{V}(g): \mathbb{C}^{(V)} \longrightarrow \mathbb{C}^{(V)} \text { and } \varrho_{E}(g): \mathbb{C}^{(E)} \longrightarrow \mathbb{C}^{(E)}
$$

the linear maps which are defined by letting $\xi_{v} \mapsto \xi_{g \cdot v}$ for all $v \in V$ and $\xi_{e} \mapsto \xi_{g \cdot e}$ for all $e \in E$. These linear maps induce algebra homomorphisms

$$
\varrho_{V}: \mathbb{C} G \longrightarrow L\left(\mathbb{C}^{(V)}\right) \text { and } \varrho_{E}: \mathbb{C} G \longrightarrow L\left(\mathbb{C}^{(E)}\right)
$$

We now assume that $X$ is a tree and fix a vertex $v_{0} \in V$. Then, using the associated bijection $\lambda: V \backslash\left\{v_{0}\right\} \rightarrow E$, we may define the linear maps

$$
p: \mathbb{C}^{(V)} \longrightarrow \mathbb{C}^{(E)} \text { and } q: \mathbb{C}^{(E)} \longrightarrow \mathbb{C}^{(V)},
$$

by letting $p\left(\xi_{v}\right)=\xi_{\lambda(v)}$ for all $v \in V \backslash\left\{v_{0}\right\}, p\left(\xi_{v_{0}}\right)=0$ and $q\left(\xi_{e}\right)=\xi_{\lambda-1}(e)$ for all $e \in E$. It is easily seen that $p \circ q=1 \in L\left(\mathbb{C}^{(E)}\right)$ and $q \circ p=1-p_{0} \in L\left(\mathbb{C}^{(V)}\right)$, 
where $p_{0} \in L\left(\mathbb{C}^{(V)}\right)$ is the projection onto the 1-dimensional subspace $\mathbb{C} \cdot \xi_{v_{0}}$, which vanishes on $\xi_{v}$ for all $v \in V \backslash\left\{v_{0}\right\}$. In particular, the linear map

$$
\widetilde{\varrho_{E}}: \mathbb{C} G \longrightarrow L\left(\mathbb{C}^{(V)}\right) \text {, }
$$

which is defined by letting $\widetilde{\varrho_{E}}(g)=q \circ \varrho_{E}(g) \circ p$ for all $g \in G$, is a homomorphism of non-unital algebras.

We say that the group $G$ acts without inversions on the tree $X$ if the automorphism induced by the element $g \in G$ on $X$ has no inversions for all $g \in G$.

Proposition 2.3. Let $G$ be a group acting on a tree $X$ without inversions, fix a vertex $v_{0} \in V$ and consider the algebra homomorphisms $\varrho_{V}$ and $\widetilde{\varrho_{E}}$ defined above. Then, for any element $g \in G$ the operator $\varrho_{V}(g)-\widetilde{\varrho_{E}}(g) \in L\left(\mathbb{C}^{(V)}\right)$ is of finite rank, whereas its trace (cf. Example 1.2) is equal to 1 if $g$ stabilizes some vertex of the tree and vanishes otherwise.

Proof. First of all, we note that $\varrho_{V}(g)\left(\xi_{v}\right)=\xi_{g \cdot v}$ for all $v \in V$ and $\widetilde{\varrho_{E}}(g)\left(\xi_{v}\right)=\xi_{v^{\prime}}$, where $v^{\prime}=\lambda^{-1}(g \cdot \lambda(v))$ for all $v \in V \backslash\left\{v_{0}\right\}$. Moreover, we have

$$
\lambda^{-1}(g \cdot \lambda(v))=g \cdot v \Longleftrightarrow g \cdot \lambda(v)=\lambda(g \cdot v)
$$

for all $v \in V \backslash\left\{v_{0}, g^{-1} \cdot v_{0}\right\}$. Hence, Proposition 2.2 (i) shows that if $g \cdot v_{0}=v_{0}$ then the operator $\varrho_{V}(g)-\widetilde{\varrho_{E}}(g)$ is equal to the projection $p_{0}$ onto the 1-dimensional subspace $\mathbb{C} \cdot \xi_{v_{0}}$, which vanishes on $\xi_{v}$ for all $v \in V \backslash\left\{v_{0}\right\}$. It follows that $\varrho_{V}(g)-\widetilde{\varrho_{E}}(g)$ is of finite rank and $\operatorname{Tr}\left[\varrho_{V}(g)-\widetilde{\varrho_{E}}(g)\right]=1$. We now assume that $g \cdot v_{0} \neq v_{0}$. In that case, we let $\left(e_{1}, \ldots, e_{n}\right)$ be the geodesic $\left[v_{0}, g^{-1} \cdot v_{0}\right]$ and consider for all $i=1, \ldots, n$ the terminal vertex $v_{i} \in V$ of $e_{i}$; in particular, $v_{n}=g^{-1} \cdot v_{0}$. Then, Proposition 2.2 (ii) implies that the operator $\varrho_{V}(g)-\widetilde{\varrho_{E}}(g)$ vanishes on $\xi_{v}$ for all $v \in V \backslash\left\{v_{0}, v_{1}, \ldots, v_{n-1}, v_{n}\right\}$; in particular, $\varrho_{V}(g)-\widetilde{\varrho_{E}}(g)$ is of finite rank. On the other hand, it is easily seen that

$$
\left(\varrho_{V}(g)-\widetilde{\varrho_{E}}(g)\right)\left(\xi_{v_{i}}\right)= \begin{cases}\xi_{g} \cdot v_{0} & \text { if } i=0, \\ \xi_{g} \cdot v_{i}-\xi_{g \cdot v_{i-1}} & \text { if } i=1, \ldots, n .\end{cases}
$$

Therefore, the final assertion in the statement of the proposition to be proved follows readily from the next lemma.

Lemma 2.4. Let $X$ be a tree and $\alpha$ an automorphism of $X$. We consider a vertex $v_{0} \in V$ such that $v^{\prime}=\alpha\left(v_{0}\right) \neq v_{0}$, and the geodesic $\left[v_{0}, v^{\prime}\right]=\left(e_{1}, \ldots, e_{n}\right)$. We denote by $v_{i}$ the terminal vertex of $e_{i}$ for all $i=1, \ldots, n$; in particular, $v_{n}=v^{\prime}$. Then:

(i) If $\alpha$ has no inversions, then $\alpha\left(v_{i}\right) \neq v_{i-1}$ for all $i=1, \ldots, n$.

(ii) If $\alpha$ fixes some vertex $v \in V$, then there is a unique $i \in\{1, \ldots, n-1\}$ such that $\alpha\left(v_{i}\right)=v_{i}$. 


\section{Actions on trees and integrality of traces}

We assume that $G$ is a group acting on a tree $X$ without inversions. We let $V$ be the set of vertices of $X$ and consider for any element $g \in G$ the fixed point set $V^{g}=\{v \in V: g \cdot v=v\}$. We also consider the subset $S \subseteq G$, consisting of those elements $g \in G$ for which the fixed point set $V^{g}$ is non-empty. In other words, $S$ consists of those group elements that stabilize some vertex of the tree, i.e. $S=\bigcup_{v \in V} \operatorname{Stab}_{v}$. Since the set $S$ is closed under conjugation, the linear map (partial augmentation)

$$
\tau_{S}: \mathbb{C} G \longrightarrow \mathbb{C},
$$

which maps any element $\sum_{g \in G} a_{g} g \in \mathbb{C} G$ onto the complex number $\sum_{g \in S} a_{g}$, is easily seen to be a trace. The trace $\tau_{S}$ maps a group element $g \in G$ onto 1 (resp. onto 0 ) if $g$ stabilizes a vertex (resp. if $g$ does not stabilize any vertex). We consider the subset $[S] \subseteq \mathfrak{C}(G)$ which consists of the conjugacy classes of the elements of $S$, i.e. we let

$$
[S]=\{[g] \in \mathfrak{C}(G): g \in S\}=\left\{[g] \in \mathfrak{C}(G): V^{g} \neq \emptyset\right\} .
$$

Then, the trace $\tau_{S}$ factors through the quotient $\mathbb{C} G /[\mathbb{C} G, \mathbb{C} G]$ as the composition

$$
\mathbb{C} G \stackrel{p}{\longrightarrow} \mathbb{C} G /[\mathbb{C} G, \mathbb{C} G] \stackrel{\overline{\tau_{S}}}{\longrightarrow} \mathbb{C} .
$$

Here, $p$ is the quotient map, whereas $\overline{\tau_{S}}$ maps any element $\sum_{[g] \in \mathbb{C}(G)} a_{[g]}[g] \in$ $\mathbb{C} G /[\mathbb{C} G, \mathbb{C} G]$ onto the complex number $\sum_{[g] \in[S]} a_{[g]}$. In view of Remark 1.1 (i), we conclude that the additive map

$$
\left(\tau_{S}\right)_{*}: K_{0}(\mathbb{C} G) \longrightarrow \mathbb{C},
$$

which is induced by the trace $\tau_{S}$, coincides with the composition

$$
K_{0}(\mathbb{C} G) \stackrel{r_{\mathrm{HS}}}{\longrightarrow} \mathbb{C} G /[\mathbb{C} G, \mathbb{C} G] \stackrel{\overline{\tau_{S}}}{\longrightarrow} \mathbb{C} .
$$

Therefore, $\left(\tau_{S}\right)_{*}$ maps any element $x \in K_{0}(\mathbb{C} G)$ with Hattori-Stallings rank $\sum_{[g] \in \mathbb{C}(G)} r_{[g]}(x)[g]$ onto the complex number $\sum_{[g] \in[S]} r_{[g]}(x)$. Since the subset $S \subseteq G$ is obviously closed under $n$-th powers for all $n \geq 1$, it follows from [8, Proposition 3.2] that $\sum_{[g] \in[S]} r_{[g]}(x) \in \mathbb{Q}$. The following result strengthens that assertion, as it states that the above rational number is, in fact, an integer.

Theorem 3.1. Let $G$ be a group acting on a tree $X$ without inversions and consider the subset $S \subseteq G$ and the additive map

$$
\left(\tau_{S}\right)_{*}: K_{0}(\mathbb{C} G) \longrightarrow \mathbb{C}
$$

defined above. Then, $\operatorname{im}\left(\tau_{S}\right)_{*}=\mathbb{Z} \subseteq \mathbb{C}$. 
Proof. Since $\tau_{S}(1)=1$, it follows that $\mathbb{Z} \subseteq \operatorname{im}\left(\tau_{S}\right)_{*}$. In order to prove the reverse inclusion, we shall use the following result.

Theorem 3.2. Let $G$ be a group acting on a tree $X$ without inversions and consider the subset $S \subseteq G$ defined above. Then, for any $x \in K_{0}(\mathbb{C} G)$ there exists a suitable element $y \in K_{0}(\mathbb{C} G)$ such that $r_{[g]}(y)=r_{[g]}(x)$ if $g \in S$ and $r_{[g]}(y)=0$ if $g \notin S$.

Proof. We fix a vertex $v_{0} \in V$ and consider the representations

$$
\varrho_{V}: \mathbb{C} G \longrightarrow L\left(\mathbb{C}^{(V)}\right) \text { and } \widetilde{\varrho_{E}}: \mathbb{C} G \longrightarrow L\left(\mathbb{C}^{(V)}\right)
$$

which were defined before the statement of Proposition 2.3. Using the Hopf algebra structure of $\mathbb{C} G$, we now define the algebra homomorphisms

$$
\sigma_{V}: \mathbb{C} G \longrightarrow L\left(\mathbb{C}^{(V)}\right) \otimes \mathbb{C} G \text { and } \widetilde{\sigma_{E}}: \mathbb{C} G \longrightarrow L\left(\mathbb{C}^{(V)}\right) \otimes \mathbb{C} G,
$$

by letting $\sigma_{V}(g)=\varrho_{V}(g) \otimes g$ and $\widetilde{\sigma_{E}}(g)=\widetilde{\varrho_{E}}(g) \otimes g$ for all $g \in G$. Then, for any $g \in G$ we have $\sigma_{V}(g)-\widetilde{\sigma_{E}}(g)=\left[\varrho_{V}(g)-\widetilde{\varrho_{E}}(g)\right] \otimes g$ and hence Proposition 2.3 implies that $\sigma_{V}(a)-\widetilde{\sigma_{E}}(a) \in \mathfrak{F} \otimes \mathbb{C} G$ for all $a \in \mathbb{C} G$, where $\mathfrak{F} \subseteq L\left(\mathbb{C}^{(V)}\right)$ is the ideal of finite rank operators on $\mathbb{C}^{(V)}$.

We also consider the trace

$$
\operatorname{Tr} \otimes p: \mathfrak{F} \otimes \mathbb{C} G \longrightarrow \mathbb{C} G /[\mathbb{C} G, \mathbb{C} G],
$$

where $\operatorname{Tr}$ is the standard trace on $\mathfrak{F}$ and $p$ the universal trace on $\mathbb{C} G$ (cf. Remark 1.1 (iii) and Example 1.2), and define the map $t$ as the composition

$$
\mathbb{C} G \stackrel{\sigma_{V}-\widetilde{\sigma_{E}}}{\longrightarrow} \mathfrak{F} \otimes \mathbb{C} G \stackrel{\operatorname{Tr} \otimes p}{\longrightarrow} \mathbb{C} G /[\mathbb{C} G, \mathbb{C} G] .
$$

Then, $t$ is a trace as well, in view of Proposition 1.3 (i). Moreover, Proposition 1.3 (ii) implies that the image of the induced additive map

$$
t_{*}: K_{0}(\mathbb{C} G) \longrightarrow \mathbb{C} G /[\mathbb{C} G, \mathbb{C} G]
$$

is contained in the image of the additive map

$$
(\operatorname{Tr} \otimes p)_{*}: K_{0}(\mathfrak{F} \otimes \mathbb{C} G) \longrightarrow \mathbb{C} G /[\mathbb{C} G, \mathbb{C} G] .
$$

Hence, in view of the identification of the latter map with the Hattori-Stallings rank map $r_{\mathrm{HS}}$ on $K_{0}(\mathbb{C} G)$ (cf. Remark 1.1 (iii) and Example 1.2), we conclude that im $t_{*} \subseteq$ im $r_{\mathrm{HS}}$.

On the other hand, Proposition 2.3 implies that the trace $t$ maps any group element $g \in G$ onto $[g]$ (resp. onto 0) if $g \in S$ (resp. if $g \notin S$ ). It follows that $t$ factors as the composition

$$
\mathbb{C} G \stackrel{p}{\longrightarrow} \mathbb{C} G /[\mathbb{C} G, \mathbb{C} G] \stackrel{\bar{t}}{\longrightarrow} \mathbb{C} G /[\mathbb{C} G, \mathbb{C} G],
$$


where $p$ is the quotient map and $\bar{t}$ maps any element $\sum_{[g] \in \mathbb{C}(G)} a_{[g]}[g]$ onto the partial sum $\sum_{[g] \in[S]} a_{[g]}[g]$. Hence, invoking Remark 1.1 (i), we conclude that the additive map $t_{*}$ coincides with the composition

$$
K_{0}(\mathbb{C} G) \stackrel{r_{\mathrm{HS}}}{\longrightarrow} \mathbb{C} G /[\mathbb{C} G, \mathbb{C} G] \stackrel{\bar{t}}{\longrightarrow} \mathbb{C} G /[\mathbb{C} G, \mathbb{C} G] .
$$

It follows that $t_{*}$ maps any element $x \in K_{0}(\mathbb{C} G)$ with Hattori-Stallings rank $\sum_{[g] \in \mathbb{C}(G)} r_{[g]}(x)[g]$ onto $\sum_{[g] \in[S]} r_{[g]}(x)[g]$. It follows that the assertion in the statement of Theorem 3.2 is equivalent to the inclusion im $t_{*} \subseteq \operatorname{im} r_{\mathrm{HS}}$, that we have already established.

Proof of Theorem 3.1 (continued). We fix a K-theory class $x \in K_{0}(\mathbb{C} G)$ and choose $y \in K_{0}(\mathbb{C} G)$ according to in the statement of Theorem 3.2. Then,

$$
\left(\tau_{S}\right)_{*}(x)=\sum_{[g] \in[S]} r_{[g]}(x)=\sum_{[g] \in \mathbb{C}(G)} r_{[g]}(y)
$$

is the homological rank of $y$; in particular, we have $\left(\tau_{S}\right)_{*}(x) \in \mathbb{Z}$.

At this point, we recall that there is a close relationship between group actions on trees on one hand and group splittings on the other. Using the notion of a graph of groups (cf. [6], [15]), this relationship can be described by the Bass-Serre theory, as follows:

(i) If $G$ is a group acting without inversions on a tree $X$, then there is a structure of a graph of groups on the quotient graph $Y=X / G$ such that the corresponding fundamental group is isomorphic to $G$.

(ii) Conversely, for any graph of groups on a connected graph $Y$ with fundamental group $G$ there is a tree $X$, the so-called universal tree of the graph, on which $G$ acts without inversions, in such a way that $X / G \simeq Y$ and the stabilizer of any vertex (resp. edge) of $X$ is a conjugate in $G$ of a vertex group (resp. edge group) of the graph of groups.

Hence, we may rephrase Theorem 3.1 as follows: Let $G$ be the fundamental group of a connected graph of groups with vertex groups $\left(G_{v}\right)_{v}$. For any vertex $v$ of the graph we regard the group $G_{v}$ as a subgroup of $G$ and define

$$
\left[G_{v}\right]=\left\{[g] \in \mathfrak{C}(G): g \in G_{v}\right\} .
$$

Then, for any element $x \in K_{0}(\mathbb{C} G)$ with Hattori-Stallings rank $\sum_{[g] \in \mathbb{C}(G)} r_{[g]}(x)[g]$ the complex number $\sum\left\{r_{[g]}(x):[g] \in \bigcup_{v}\left[G_{v}\right]\right\}$ is, in fact, an integer.

In particular, we obtain the following two results concerning amalgamated free products and $\mathrm{HNN}$ extensions: 
Corollary 3.3. Let $G=A \star_{H} B$ be the amalgamated free product of two groups $A$ and $B$ along a common subgroup $H$ of theirs and consider an element $x \in K_{0}(\mathbb{C} G)$ with Hattori-Stallings rank $\sum_{[g] \in \mathbb{C}(G)} r_{[g]}(x)[g]$. We view A and $B$ as subgroups of $G$ and define

$$
[A]=\{[g] \in \mathfrak{C}(G): g \in A\} \text { and }[B]=\{[g] \in \mathfrak{C}(G): g \in B\} .
$$

Then, the complex number $\sum_{[g] \in[A] \cup[B]} r_{[g]}(x)$ is, in fact, an integer.

Proof. Let $Y$ be the graph consisting of an edge $e$ and two distinct vertices $v=o(e)$ and $v^{\prime}=t(e)$. Then, the result follows from Theorem 3.1, in view of the discussion above, by considering the graph of groups on $Y$ which is given by letting $G_{e}=H$, $G_{v}=A$ and $G_{v^{\prime}}=B$ with homomorphisms $G_{e} \rightarrow G_{o(v)}$ and $G_{e} \rightarrow G_{t(e)}$ the inclusion maps of $H$ into $A$ and $B$ respectively.

Corollary 3.4. Let $A$ be a group, $H \subseteq A$ a subgroup and $\varphi: H \rightarrow A$ a monomorphism. We consider the corresponding HNN extension $G=A \star_{\varphi}$ and let $x \in$ $K_{0}(\mathbb{C} G)$ be an element with Hattori-Stallings rank $\sum_{[g] \in \mathbb{E}(G)} r_{[g]}(x)[g]$. We view $A$ as a subgroup of $G$ and define

$$
[A]=\{[g] \in \mathfrak{C}(G): g \in A\} .
$$

Then, the complex number $\sum_{[g] \in[A]} r_{[g]}(x)$ is, in fact, an integer.

Proof. Let $Y$ be the graph consisting of an edge $e$ and a vertex $v=o(e)=t(e)$. Then, the result follows from Theorem 3.1, in view of the discussion above, by considering the graph of groups on $Y$ which is given by letting $G_{e}=H, G_{v}=A$ with homomorphisms $G_{e} \rightarrow G_{o(e)}$ and $G_{e} \rightarrow G_{t(e)}$ the inclusion map of $H$ into $A$ and $\varphi: H \rightarrow A$ respectively.

Remark 3.5. The result of Corollary 3.3 admits an alternative homological proof, if the group $H$ therein is trivial. Indeed, let $G=A \star B$ be the free product of two groups $A, B$ and consider an element $g \in G$ which is not conjugate to any element of $A$ nor $B$, i.e. an element $g \in G$ for which $[g] \notin[A] \cup[B]$. Then, the centralizer $C_{g}$ of $g$ in $G$ is easily seen to be infinite cyclic; this can be proved, for example, by invoking the Bass-Serre theory of groups acting on trees. In particular, the quotient group $N_{g}=C_{g} /\langle g\rangle$ is finite and hence one may use the Connes-Karoubi character map from $K_{0}(\mathbb{C} G)$ to the second cyclic homology group of the group algebra $\mathbb{C} G$, in order to show that the coefficient $r_{[g]}(x)$ of the Hattori-Stallings rank $r_{\mathrm{HS}}(x)$ of any element $x \in K_{0}(\mathbb{C} G)$ vanishes (cf. [7]). In particular, for any $x \in K_{0}(\mathbb{C} G)$ we have $\sum_{[g] \in[A] \cup[B]} r_{[g]}(x)=\sum_{[g] \in \mathbb{C}(G)} r_{[g]}(x)$. Since the right-hand side of the latter equality is the homological rank of $x$, we conclude that $\sum_{[g] \in[A] \cup[B]} r_{[g]}(x) \in \mathbb{Z}$. 
On the other hand, if $G=A \star B$ then the additive map

$$
K_{0}(\mathbb{C} A) \oplus K_{0}(\mathbb{C} B) \longrightarrow K_{0}(\mathbb{C} G),
$$

which is induced by the inclusions of $A$ and $B$ into $G$, is surjective; this follows from the discussion following [17, Corollary 11.5]. As explained in the Introduction, the surjectivity of the above map provides yet another proof of Corollary 3.3 (in the case where $H=1$ ).

\section{Group actions on trees with finite $S$}

Our goal in this final section is to examine the extent to which Theorem 3.1 can be generalized to an integrality result concerning a trace defined on the reduced $C^{*}$ algebra of a group. Unfortunately, it will turn out that our approach does not lead to any really new results in that direction.

First of all, we recall that the group $G$ acts on the Hilbert space $\ell^{2} G$ by left translations and denote by

$$
L: \mathbb{C} G \longrightarrow \mathfrak{B}\left(\ell^{2} G\right)
$$

the induced algebra homomorphism. Then, $L$ is injective, its image $L(\mathbb{C} G)$ is a selfadjoint subalgebra of $\mathfrak{B}\left(\ell^{2} G\right)$ and the reduced $C^{*}$-algebra $C_{r}^{*} G$ of $G$ is the operator norm closure of $L(\mathbb{C} G)$ in $\mathfrak{B}\left(\ell^{2} G\right)$. The linear functional

$$
\tau: C_{r}^{*} G \longrightarrow \mathbb{C},
$$

which is defined by letting $\tau(a)=\left\langle a\left(\delta_{1}\right), \delta_{1}\right\rangle$ for all $a \in C_{r}^{*} G$, is a continuous positive faithful and normalized trace, which is referred to as the canonical trace on $C_{r}^{*} G$. (Here, we denote by $\left(\delta_{g}\right)_{g}$ the standard orthonormal basis of $\ell^{2} G$.) For later use, we note that for any element $g \in G$ the linear map $a \mapsto \tau\left(L(g)^{*} a\right), a \in$ $C_{r}^{*} G$, restricts to the subspace $\mathbb{C} G \simeq L(\mathbb{C} G)$ to the linear map $\sum_{h \in G} a_{h} h \mapsto a_{g}$, $\sum_{h \in G} a_{h} h \in \mathbb{C} G$.

In order to extend the trace $\tau_{S}$ on the group algebra $\mathbb{C} G$, which was defined in the beginning of $\S 3$, to a trace on $C_{r}^{*} G$, we shall make the following assumption: The group $G$ acts without inversions on a tree $X$ in such a way that the subset $S=\bigcup_{v \in V} \operatorname{Stab}_{v}$ of $G$, which consists of those group elements that stabilize a vertex, is finite. We note that, under this assumption, the trace $\tau_{S}$ on $\mathbb{C} G \simeq L(\mathbb{C} G)$ extends to a continuous trace

$$
\tau_{S}: C_{r}^{*} G \longrightarrow \mathbb{C}
$$

by letting $\tau_{S}(a)=\sum_{g \in S} \tau\left(L(g)^{*} a\right)$ for all $a \in C_{r}^{*} G$. Indeed, the set $S$ being finite, $\tau_{S}$ is a continuous linear functional on $C_{r}^{*} G$. In view of the remark made above, that 
linear functional restricts to the subspace $\mathbb{C} G \simeq L(\mathbb{C} G)$ to the trace $\tau_{S}$ on $\mathbb{C} G$. It follows by continuity that $\tau_{S}$ satisfies the trace property on $C_{r}^{*} G$ as well. Since the set $S$ is obviously closed under inverses, we also have $\tau_{S}(a)=\sum_{g \in S} \tau(L(g) a)$ for all $a \in C_{r}^{*} G$.

It turns out that the finiteness assumption on $S$ places some severe restrictions on the group $G$. In fact, we shall prove that $S$ must be a normal subgroup of $G$ such that the quotient $G / S$ is free. Then, the integrality of the trace $\tau_{S}$ on $C_{r}^{*} G$ will be an immediate consequence of Connes' result [5, §IV.5] that free groups satisfy the integrality of the trace conjecture.

Let us consider the subset (normal subgroup) $G_{f} \subseteq G$ consisting of those elements that have only finitely many conjugates; in other words, we let

$$
G_{f}=\{g \in G: \text { the conjugacy class }[g] \text { is finite }\} .
$$

We recall that a group is 2-ended if and only if it has an infinite cyclic subgroup of finite index (cf. [6, Chapter IV, Theorem 6.12]).

Proposition 4.1. Let $G$ be a group acting without inversions on a tree $X$, in such a way that the subset $S=\bigcup_{v \in V} \operatorname{Stab}_{v}$ of $G$ is finite. Then:

(i) The stabilizer subgroup $\mathrm{Stab}_{v}$ is a finite subgroup of $G_{f}$ for all $v \in V$.

(ii) $S=\{g \in G$ : the order of $g$ is finite $\} \subseteq G_{f}$.

(iii) The group $G$ has a free subgroup of finite index.

(iv) If $G$ is not 2-ended, then $S=G_{f}$ and the quotient group $G / G_{f}$ is free.

(v) If $G$ is 2-ended, then $S$ is a normal subgroup of $G$ and the quotient group $G / S$ is infinite cyclic.

Proof. (i) Let us fix a vertex $v \in V$. Then, the finiteness of $\mathrm{Stab}_{v}$ is clear, since $\mathrm{Stab}_{v} \subseteq S$. On the other hand, $S$ is closed under conjugation and hence for any $g \in \operatorname{Stab}_{v}$ the conjugacy class $[g]$ is contained in $S$; in particular, $[g]$ is a finite set, i.e. $g \in G_{f}$.

(ii) Since $S=\bigcup_{v \in V} \operatorname{Stab}_{v}$ is a union of finite subgroups of $G_{f}$ (in view of (i) above), it is contained itself in $G_{f}$ and consists of elements of finite order. On the other hand, any torsion element $g \in G$ acts on the tree $X$ by fixing some vertex (cf. [15, Chapitre I, Exemple 6.3.1]); hence, $g \in S$. We conclude that $S=\{g \in G$ : the order of $g$ is finite\}.

(iii) Since the orders of the stabilizer subgroups $\operatorname{Stab}_{v}, v \in V$, are obviously bounded by some integer, the result follows from [6, Chapter IV, Theorem 1.6].

(iv) We fix a free normal subgroup $N \subseteq G$ of finite index; such a subgroup exists, in view of (iii) above. Since the group $G$ is not 2-ended, the free group $N$ is not infinite cyclic. Hence, all non-identity elements of $N$ have infinitely many conjugates in $N$ 
and, a fortiori, in $G$; in particular, $N \cap G_{f}=1$. It follows that $G_{f}$ embeds in $G / N$ and hence $G_{f}$ is a finite group. As such, $G_{f}$ is contained in the subset of torsion elements of $G$ and hence $G_{f}=S$, in view of (ii) above. Since the free group $N$ embeds as a subgroup of finite index in $G / G_{f}$, we may invoke [6, Chapter IV, Theorem 1.6] once again, in order to conclude that there is a tree $T$ on which $G / G_{f}$ acts without inversions, in such a way that the vertex stabilizer subgroups are finite (and have orders bounded by some integer). On the other hand, since $G_{f}$ coincides with the subset of torsion elements of $G$, the group $G / G_{f}$ is easily seen to be torsionfree. It follows that the action of $G / G_{f}$ on the tree $T$ must be free. Hence, invoking [15, Chapitre I, §3.3], we conclude that the group $G / G_{f}$ is free.

(v) It is well known that a 2-ended group $G$ admits a surjective homomorphism with finite kernel onto the infinite cyclic group $\mathbb{Z}$ or else onto the infinite dihedral group $D_{\infty}$. The latter case cannot occur, since $D_{\infty}$ has infinitely many elements of finite order, whereas the corresponding set for $G$ is finite (in view of (ii) above). Therefore, there is a finite normal subgroup $H$ of $G$ such that $G / H \simeq \mathbb{Z}$. It is now clear that $H$ coincides with the set of elements of finite order in $G$ and hence the proof is finished.

Let us now consider the group $G$ which acts without inversions on a tree $X$, in such a way that the subset $S \subseteq G$ consisting of those group elements that stabilize a vertex is finite. Then, it follows from Proposition 4.1 that $S$ is a finite normal subgroup of $G$, whereas the quotient group $\bar{G}=G / S$ is free. In view of the finiteness of $S$, the quotient map $G \rightarrow \bar{G}$ induces an algebra homomorphism

$$
\pi_{0}: \mathbb{C} G \longrightarrow \mathbb{C} \bar{G},
$$

which can be extended to a $*$-algebra homomorphism

$$
\pi: C_{r}^{*} G \longrightarrow C_{r}^{*} \bar{G} .
$$

We note that the trace $\tau_{S}$ on $C_{r}^{*} G$, which was defined in the beginning of this section, coincides with the composition

$$
C_{r}^{*} G \stackrel{\pi}{\longrightarrow} C_{r}^{*} \bar{G} \stackrel{\bar{\tau}}{\longrightarrow} \mathbb{C},
$$

where $\bar{\tau}$ is the canonical trace on $C_{r}^{*} \bar{G}$. In order to verify this latter assertion, it suffices (by continuity) to show that the trace $\tau_{S}$ on $\mathbb{C} G$, which was defined in the beginning of $\S 3$, coincides with the composition

$$
\mathbb{C} G \stackrel{\pi_{0}}{\longrightarrow} \mathbb{C} \bar{G} \stackrel{\bar{\tau}}{\longrightarrow} \mathbb{C},
$$

where $\bar{\tau}$ is the linear trace on $\mathbb{C} \bar{G}$, which maps $\overline{1} \in \bar{G}$ onto 1 and any element $\bar{g} \in \bar{G} \backslash\{\overline{1}\}$ onto 0 . But this is clear, in view of the definitions. Invoking now 
Remark 1.1 (ii), we conclude that the additive map

$$
\left(\tau_{S}\right)_{*}: K_{0}\left(C_{r}^{*} G\right) \longrightarrow \mathbb{C},
$$

which is induced by the trace $\tau_{S}$ on $C_{r}^{*} G$, coincides with the composition

$$
K_{0}\left(C_{r}^{*} G\right) \stackrel{K_{0}(\pi)}{\longrightarrow} K_{0}\left(C_{r}^{*} \bar{G}\right) \stackrel{\bar{\tau}_{*}}{\longrightarrow} \mathbb{C},
$$

where $\bar{\tau}_{*}$ is the additive map induced by the canonical trace $\bar{\tau}$ on $C_{r}^{*} \bar{G}$. Therefore, the group $\bar{G}$ being free, we may invoke Connes' result [5, §IV.5] that free groups satisfy the integrality of the trace conjecture, in order to conclude that the image of the additive map (4) is the group $\mathbb{Z}$ of integers.

\section{References}

[1] A. Bartels, W. Lück, Isomorphism conjecture for homotopy K-theory and groups acting on trees. J. Pure Appl. Algebra 205 (2006), 660-696. Zbl 1093.19002 MR 2210223

[2] H. Bass, Euler characteristics and characters of discrete groups. Invent. Math. 35 (1976), 155-196. Zbl 0365.20008 MR 0432781

[3] P. Baum, A. Connes, Geometric K-theory for Lie groups and foliations. Enseign. Math. (2) 46 (1-2) (2000), 3-42. Zbl 0985.46042 MR 1769535

[4] K. S. Brown, Cohomology of groups. Grad. Texts Math. 87, Springer-Verlag, New York 1982. Zbl 0584.20036 MR 0672956

[5] A. Connes, Noncommutative geometry. Academic Press Inc., San Diego 1994. Zbl 0818.46076 MR 1303779

[6] W. Dicks, M. J. Dunwoody, Groups acting on graphs. Cambridge University Press, Cambridge 1989. Zbl 0665.20001 MR 1001965

[7] B. Eckmann, Cyclic homology of groups and the Bass conjecture. Comment. Math. Helv. 61 (1986), 193-202. Zbl 0613.20034 MR 0856086

[8] I. Emmanouil, Traces and idempotents in group algebras. Math. Z. 245 (2003), 293-307. Zbl 1039.16028 MR 2013502

[9] I. Emmanouil, Idempotent matrices over complex group algebras. Universitext, SpringerVerlag, New York 2006. Zbl 1093.16023 MR 2184145

[10] W. Lück, The relation bewtween the Baum-Connes conjecture and the trace conjecture. Invent. Math. 149 (2002), 123-152. Zbl 1035.19003 MR 1914619

[11] W. Lück, $L^{2}$-invariants: theory and applications to geometry and K-theory. Ergeb. Math. Grenzgeb. 44, Springer-Verlag, Berlin 2002. Zbl 1009.55001 MR 1926649

[12] H. Oyono-Oyono, Baum-Connes conjecture and group actions on trees. K-Theory 24 (2001), 115-134. Zbl 1008.19001 MR 1869625

[13] J. Rosenberg, Algebraic K-theory and its applications. Grad. Texts Math. 147, SpringerVerlag, New York 1994. Zbl 0801.19001 MR 1282290 
[14] R. Roy, The trace conjecture - a counterexample. K-Theory 17 (1999), 209-213. Zbl 0939.19003 MR 1703309

[15] J. P. Serre, Arbres, amalgames, SL 2. Astérisque 46 (1977). Zbl 0369.20013 MR 0476875

[16] A. Valette, The conjecture of idempotents: A survey of the $C^{*}$-algebraic approach. Bull. Soc. Math. Belg. 41 (1989), 485-521. Zbl 0692.46063 MR 1037649

[17] F. Waldhausen, Algebraic $K$-theory of generalized free products. III, IV. Ann. of Math. 108 (1978), 205-256. Zbl 0407.18009 MR 0498808

[18] A. E. Zalesskii, On a problem of Kaplansky. Dokl. Akad. Nauk SSSR 203 (1972), 749-751; English transl. Soviet. Math. 13 (1972), 449-452. Zbl 0257.16010 MR 297895

Received March 20, 2007

Ioannis Emmanouil, Department of Mathematics, University of Athens, Athens 15784, Greece

E-mail: emmanoui@math.uoa.gr 